\title{
Acomodación a la enfermedad, percepción de carga y malestar emocional en familiares de pacientes con un trastorno alimentario.
}

\section{Accommodation to illness, burden perception and emotional distress in family members of patients with an eating disorder.}

Fecha de recepción: 10-07-2018

Fecha de aceptación: 12-07-2018

\author{
Brígida Pérez Pareja \\ Universidad Cardenal Herrera CEU. Centro CREA. \\ Yolanda Quiles Marcos \\ Universidad Miguel Hernández. Centro CREA \\ María José Quiles Sebastián \\ Universidad Miguel Hernández. Centro CREA \\ Lidia Pamies Aubalat \\ Universidad Católica San Antonio de Murcia
}

\section{resumen/alsstract:}

Los trastornos alimentarios no solo afectan a la persona que los sufre sino también a sus familiares. La literatura señala que es frecuente que los familiares de estas pacientes presenten una elevada percepción de carga, malestar emocional y acomodación al síntoma. Estas variables se han relacionado con el pronóstico y evolución de estas pacientes. El objetivo del presente trabajo fue evaluar y describir la acomodación a la enfermedad, la percepción de carga y el malestar emocional en 93 familiares de pacientes diagnosticados con un trastorno alimentario, así como analizar las relaciones entre estas variables. Los resultados mostraron diferencias significativas entre hombres y mujeres en la percepción de carga, la acomodación y el malestar emocional, presentando las familiares mujeres mayores puntuaciones en estas variables. Estas variables presentaron relaciones positivas y significativas entre ellas. El aislamiento social predijo un $46.5 \%$ de la varianza del malestar emocional de los cuidadores. Estos resultados sugieren la importancia de apoyar a los familiares y diseñar intervenciones específicas para ellos.

Eating disorders (ED) not only affect the person who suffers but also their family members. The literature indicates that the relatives of these patients frequently have a high perception of burden, emotional distress and accommodation to the symptom. These variables have been related to the prognosis and evolution of these patients. The objective of the present work was to evaluate and describe the accommodation to the disease, the perception of burden and emotional distress in 93 relatives of ED patients, as well as to analyze the relationships between these variables. The results showed significant differences between men and women in burden perception, accommodation and emotional distress, female relatives showed higher scores on these variables. These variables showed positive and significant relationships among them. Social isolation predicted $46.5 \%$ of the variance of the emotional distress of the caregivers. These results suggest the importance of supporting family members and designing specific interventions for them.

\section{palabras clave/keywords:}

Trastornos Alimentarios, Cuidadores, Familia, Malestar Emocional, Acomodación a la enfermedad, Percepción de carga Eating disorders, accomodation to illness, burden perception, Emotional distress, Caregiver, Family. 


\section{Introducción.}

Los Trastornos Alimentarios (TA) hacen referencia a alteraciones psicológicas, cuya característica principal es la presencia de graves anormalidades en el comportamiento alimentario, que repercuten sobre la salud de las personas que la padecen, dando lugar a alteraciones físicas, conductuales y psicológicas (Calvo, 2002). Son una de las psicopatologías más frecuentes en mujeres adolescentes, y despiertan un interés social, psicológico y científico, debido a la gravedad de sus síntomas, su elevada resistencia al tratamiento, el riesgo de recaídas, así como el alto grado de comorbilidad y mortalidad que presentan (Birmingham, Su, Hlynsky, Goldner y Gao, 2005; Strober, 2004).

Los TA no solo afectan a la persona que lo sufre sino también a su ámbito familiar. Hay que tener en cuenta que el TA aparece normalmente en la adolescencia, cuando aún están bajo el cuidado de los padres, viviendo en el hogar familiar, por lo que los cambios que ocurren en la paciente, afectan y tienen impacto en el resto de miembros familiares, así como en todos los aspectos de la vida familiar. Además, aunque las pacientes sean adultas, son dependientes de la familia y necesitan apoyo para poder cubrir sus necesidades a nivel económico, instrumental y emocional, por lo que también repercuten e interfieren en el curso de la vida familiar (Cottee-Lane, Pistrang y Bryant-Waugh, 2004; Highet, Thompson y King, 2005; Hjern, Lindberg y Lindblad, 2006; Whitney et al., 2005; Winn et al., 2004).

La literatura señala que es frecuente encontrar en los familiares de estas pacientes la sensación de carga, entendida como el cúmulo de problemas, dificultades y situaciones adversas que tienen que afrontar, así como todas las consecuencias físicas, sociales y emocionales que sufren como consecuencia de su rol como cuidadores (Padierna et al., 2013). Esta percepción de carga envuelve al cuidador en sentimientos de culpa e impotencia, estrés y malestar psicológico y emocional, encontrando con frecuencia en los cuidadores sintomatología depresiva y ansiosa (Perkins, Winn, Murray, Murphy y Schmidt, 2004; Tierney, 2005; Whitney et al., 2005; Zabala et al., 2009).

Este malestar emocional del familiar puede estar relacionado con la baja conciencia de enfermedad de las pacientes, así como su frecuente rechazo a recibir tratamiento. Cuando la paciente rechaza recibir tratamiento, incluso cuando está en bajo peso y el riesgo vital es muy presente, se puede generar mucha fricción en la relación entre ella y el cuidador (Tan, Hope, Stewart y Fitzpatrick, 2003). Dicha situación potencia el malestar de los cuidadores, debido a la preocupación que sienten por su familiar, ya que resulta frustrante ver como la persona con el problema no quiere recibir ayuda ni atención, negando la severidad de sus síntomas o de su TA, mientras que por otro lado, los cuidadores perciben los síntomas graves que las pacientes experimentan y que ponen en peligro y amenazan su vida (Treasure et al., 2007).

La percepción que de los síntomas del TA tenga el familiar va a determinar su actitud ante el problema. En ocasiones, las familias con sus comportamientos pueden reforzar de un modo involuntario los síntomas de las pacientes, es lo que se conoce con el término acomodación. Por ejemplo, existen familias que dan mucho espacio y atención a la enfermedad, aceptando las conductas del TA, llegando incluso a dominar la vida familiar, resultando ser "permisi- 
vos" con los síntomas y "acomodándose" a los mismos. Ejemplos de acomodación al síntoma podría ser: aceptar las reglas en torno a la comida impuesta por el trastorno, permitir conductas inadecuadas como la práctica excesiva de ejercicio, vómitos o el uso de laxantes o diuréticos, o aceptar los comportamientos obsesivos de las pacientes sobre el control de todos los componentes de la casa, la comida y su funcionamiento (Treasure et al., 2007). La literatura pone de manifiesto que en las familias puede darse una mayor acomodación a los síntomas del TA cuando los familiares se sienten avergonzados, culpables o con miedo al TA, y que por tanto las intervenciones familiares necesitan abordar las emociones del cuidador, ya que estas reacciones emocionales pueden aumentar la acomodación a la enfermedad (Stillar et al., 2016).

Esta acomodación al síntoma se ha relacionado con el malestar emocional de las pacientes, así en un estudio realizado por Salerno et al. (2016), donde se investigó la relación entre los comportamientos ineficaces en torno a la enfermedad en 54 padres y 54 madres de pacientes con un TA, se mostró que el malestar psicológico de las pacientes era significativamente peor, cuando los padres o las madres puntuaban alto en la variable de acomodación a la enfermedad, y que la recuperación de ellas era peor cuando ambos cuidadores presentaban alta acomodación a los síntomas del TA. Este estudio también reveló que cuando en una misma pareja existían diferencias en la acomodación a la enfermedad, no repercutía directamente en la recuperación de la paciente, pero sin embargo, cuando ambos presentaban un nivel de acomodación alto, si influía en la recuperación, ya que reforzaba los síntomas en ellos. Este estudio mostró que cuando ambos padres, o solo la madre presentaban una menor acomodación, los resultados en las pacientes eran más positivos que cuando era solo el padre el que presentaba una menor acomodación en la familia. Rhind et al. (2016), mostraron que los padres presentaban menor acomodación a los síntomas del TA frente a las madres, aunque eran ellas en mayor proporción las cuidadoras principales de las pacientes.

Dada la importancia que tiene tanto la percepción de carga del cuidador, el malestar emocional y la acomodación al síntoma en el bienestar de los familiares y en la evolución de la enfermedad, el objetivo del presente trabajo fue evaluar y describir la acomodación a la enfermedad, la percepción de carga y el malestar emocional en familiares de pacientes con un TA, así como analizar las relaciones entre estas variables.

\section{Material y métodos.}

\section{Participantes.}

Se reclutó una muestra de 93 cuidadores de pacientes diagnosticados con un TA, atendidos en la Unidad Hospitalaria de Trastornos Alimentarios (UHTA) del Hospital Universitario de San Juan (Alicante). El 32.3\% fueron hombres, y el 67.7\% mujeres, con edades comprendidas entre los 23 y los 68 años $(M=47.40 ; D T=7.12)$ donde la mayor franja de cuidadores se situaban entre los 40 y los 50 años. Eran cuidadores en mayor medida de pacientes mujeres $87.1 \%$ y la edad de las pacientes a las que cuidaban estaba comprendida entre los 10 y los 26 años $(M=17.41 ; D T=3.76)$, encontrando la mayor proporción de pacientes en edades ente los 15 y los 20 años. En relación al diagnóstico, el 76.4\% eran cuidadores de pacientes diagnosticados con Anorexia Nerviosa (AN), el 4.3\% eran cuidadores de pacien- 
tes con Bulimia Nerviosa (BN), siendo el resto cuidadores de pacientes diagnosticados con un trastorno de la conducta alimentaria no especificado (TCANE). En relación al tiempo de evolución del problema, oscilaba entre 3 meses y 5 años, y la mayor proporción de cuidadores se situaba alrededor de los 12 meses de evolución del trastorno. El $90.3 \%$ de los cuidadores convivían con los pacientes.

\section{Procedimiento.}

En primer lugar se llevó a cabo una reunión con el equipo de psicología y psiquiatría de la UHTA, donde se les contaron los objetivos de la investigación. El equipo de la UHTA facilitó el contacto con 96 familias de pacientes con un TA atendidos en ese momento tanto a nivel ambulatorio (cuyo ingreso había tenido lugar en los últimos dos años), como de hospitalización en la unidad. Los criterios de inclusión/exclusión fueron los siguientes:

- Tener un paciente diagnosticado con TA, menor de 26 años.

- Que dicho paciente no presentase comorbilidad con trastornos de personalidad.

- Ser cuidador directo del paciente.

A cada familia se les envió una carta de presentación del proyecto de investigación y posteriormente fueron llamadas para informarles de los objetivos y de las condiciones de participación. Aquellos que manifestaron su interés por participar, se les citó en la UHTA y se firmó el consentimiento informado, así como se administró la batería de cuestionarios. Todas las familias decidieron participar de forma voluntaria y fueron informadas de la confidencialidad de sus datos.

De las 76 familias citadas asistieron 74, que sumaban 103 cuidadores. Una vez cribados los cuestionarios, el número final de cuidadores que participaron fue de 93.

\section{Instrumentos.}

Cuestionario de Salud General, GHQ-12 (General Health Questionnaire) adaptación española de Rocha et al. (2011). Cuestionario de salud general de Golberg en su versión de doce ítems. Se utilizó para evaluar el malestar emocional en los cuidadores. Consta de 12 ítems con un formato de respuesta de cuatro alternativas. Permite hacer un cribado poblacional detectando la prevalencia de casos probables de morbilidad psiquiátrica o de padecimiento psicológico en la población, explorando su estado habitual durante el último mes respecto al estado habitual de la persona. La puntuación máxima del cuestionario 12 y la mínima 0 . En este estudio se obtuvo un alfa de Chronbach de .90 .

Escala de impacto de los síntomas de los trastornos alimentarios, EDSIS-S, adaptación española de Carral-Fernández, Sepúlveda, Gómez, Graell y Treasure (2013). Evaluamos la percepción de carga considerando el impacto que la enfermedad tiene en el cuidador. Consta de 24 ítems que forman 4 escalas y ofrece una puntuación total. El alfa de Chronbach en la adaptación española es de .92. En este estudio el alfa de Chronbach total fue de .90, para la subescala de impacto nutricional .84 , para la de culpa .88 , para la de conductas desreguladas .78, y para la de aislamiento social .75 . 
Tabla 1.- Estadísticos descriptivos de las subescalas en la percepción de carga

\begin{tabular}{cccccc}
\hline & EDSIS 1 & EDSIS 2 & EDSIS 3 & EDSIS. 4 & EDSIS T. \\
\hline $\mathrm{N}$ & 91 & 92 & 90 & 90 & 89 \\
Media & 12.34 & 8.53 & 4.57 & 7.07 & 32.36 \\
$D T$ & 7.11 & 5.64 & 4.84 & 4.57 & 17.58 \\
Mediana & 12.00 & 8.00 & 3.00 & 7.00 & 30.00 \\
Máximo & 28 & 20 & 21 & 19 & 80 \\
Mínimo & 0 & 0 & 0 & 0 & 0
\end{tabular}

EDSIS= Escala de impacto de los síntomas de los trastornos alimentarios; EDSIS 1= Impacto nutricional; EDSIS 2= Culpa; EDSIS 3= Conductas desrreguladas; EDSIS 4= Aislamiento Social, EDSIS T: Puntuación total.

Escala de acomodación a los síntomas de enfermedad, EAISA, validación española de Quiles, Quiles, Pamies, Sepúlveda y Treasure (2016). Evalúa la acomodación a los síntomas por parte de la familia a la enfermedad. Consta de un total de 33 ítems que evalúan 5 escalas: evitación y modificación de las rutinas, búsqueda de seguridad, rituales con la comida, control de la familia y hacer la vista gorda. El alfa de Chronbach osciló entre .79 y .89. En nuestro estudio, el alfa de Chronbach total fue de .91, y en las diferentes escalas osciló entre .90 у .71 .

\section{Análisis estadístico.}

Para el tratamiento de los datos y los análisis estadísticos, se utilizó el paquete estadístico SPSS (Versión 20.0), y los análisis que realizamos fueron los siguientes:

- Datos descriptivos de las variables utilizadas en el estudio.

- Diferencias entre medias utilizando U de Mann-Whitney como prueba no paramétrica para dos muestras independientes, y ANOVA de un factor de Kruskal-Wallis como prueba no paramétrica de $\mathrm{K}$ muestras independientes, medias repetidas y procedimiento Bonferronni para comparaciones múltiples.

- Análisis de Relaciones entre variables, aplicamos la correlación producto momento de Pearson.

- Análisis de Predicciones entre variables, utilizamos el análisis de regresión aplicando el método de pasos sucesivos (Stepwise) para evaluar el poder predictivo de cada variable. 


\section{Resultados.}

Respecto a la percepción de carga, en la tabla 1 se muestran los estadísticos descriptivos de las diferentes subescalas de esta variable.

Los análisis no mostraron diferencias significativas en esta variable en relación al tiempo de evolución del problema, el tipo de parentesco, el diagnóstico del paciente, y la convivencia con él.

Sin embargo, respecto al género del cuidador, los análisis mostraron diferencias estadísticamente significativas en relación al impacto nutricional, la culpa, las conductas desreguladas, así como la puntuación total de la escala, donde las mujeres presentaban puntuaciones medias superiores frente al grupo de los hombres (Ver tabla 2).

En relación a la edad del cuidador, los análisis mostraron diferencias estadísticamente significativas en relación a la culpa, entre los cuidadores con edades comprendidas entre 41 y 50 años y los cuidadores con más de 60 años $(\mathrm{F}=3.32, \mathrm{p} \leq .001)$.

En la tabla 3 se muestran los estadísticos descriptivos de las diferentes subescalas de la acomodación a la enfermedad. Los análisis no mostraron diferencias significativas en relación al tiempo de evolución del problema, ni en la convivencia con el paciente.

En función del género del cuidador, los análisis mostraron diferencias estadísticamente significativas en la subescala de búsqueda de seguridad, donde las mujeres presentaban puntuaciones medias superiores $(M=10.82 ; D T=7.63)$ que el grupo de los hombres $(M=6.97$; $D T=10.82)($ Ver tabla 4$)$.

Tabla 2.- Análisis de diferencias en percepción de carga y género

\begin{tabular}{cllclc}
\hline & GÉNERO & $\mathrm{N}$ & MEDIA & DT & $t$ \\
\hline \multirow{2}{*}{ IMPACTO } & Hombre & 29 & 10.03 & 6.52 & $.034^{* *}$ \\
NUTRICIONAL & Mujer & 62 & 13.42 & 7.16 & \\
\hline \multirow{2}{*}{ CULPA } & Hombre & 30 & 6.80 & 5.04 & $.040^{*}$ \\
& Mujer & 62 & 9.37 & 5.76 & \\
\hline CONDUCTAS & Hombre & 29 & 3.41 & 3.06 & $.030^{*}$ \\
DESRREGULADAS & Mujer & 61 & 5.11 & 5.43 & .62 \\
\hline AISLAMIENTO & Hombre & 29 & 5.55 & 3.64 & \\
SOCIAL & Mujer & 61 & 7.79 & 4.82 & $.013 * *$ \\
\hline TOTAL & Hombre & 29 & 25.79 & 12.84 & \\
\hline
\end{tabular}

$(* \mathrm{p} \leq 0.05 ; * * \mathrm{p} \leq 0.01 ; * * * \mathrm{p} \leq 0.001)$ 
Tabla 3.- Estadísticos descriptivos de las subescalas de la acomodación a los síntomas de enfermedad

\begin{tabular}{ccccccc}
\hline & EAISA & EAISA & EAISA & EAISA & EAISA & EAISA T \\
& 1 & 2 & 3 & 4 & 5 & \\
\hline $\mathrm{N}$ & 86 & 86 & 86 & 86 & 91 & 86 \\
Media & 17.34 & 9.52 & 4.06 & 7.12 & 2.09 & 40.14 \\
$D T$ & 9.66 & 7.27 & 6.07 & 4.45 & 3.23 & 22.86 \\
Mediana & 18.00 & 8.00 & 1.00 & 7.00 & 1.00 & 38.50 \\
Máximo & 44 & 28 & 27 & 16 & 16 & 97 \\
Mínimo & 0.00 & 0.00 & 0.00 & 0.00 & 0.00 & 0.00 \\
\hline
\end{tabular}

EAISA= Escala de acomodación a los síntomas de enfermedad EAISA 1= Evitación y modificación de la conducta; EAISA 2= Búsqueda de seguridad; EAISA 3= Rituales de comida; EAISA 4= Control de la familia; EAISA 5= Hacer la vista gorda; EAISA T= Puntuación total.

Tabla 4.- Acomodación a los síntomas de enfermedad y género del cuidador

\begin{tabular}{|c|c|c|c|c|c|c|}
\hline & GÉNERO & $\mathrm{N}$ & MEDIA & $D T$ & $p$ & $d$ \\
\hline \multirow{2}{*}{$\begin{array}{c}\text { Evitación y } \\
\text { modificación de } \\
\text { la conducta }\end{array}$} & Hombres & 29 & 15.38 & 9.54 & \multirow[b]{2}{*}{.182} & \multirow[b]{2}{*}{-0.30} \\
\hline & Mujeres & 57 & 18.33 & 9.65 & & \\
\hline \multirow{2}{*}{$\begin{array}{c}\text { Búsqueda de } \\
\text { seguridad }\end{array}$} & Hombres & 29 & 6.97 & 5.80 & \multirow{2}{*}{$.019 * *$} & \multirow{2}{*}{-0.54} \\
\hline & Mujeres & 57 & 10.82 & 7.63 & & \\
\hline \multirow{2}{*}{$\begin{array}{c}\text { Rituales de } \\
\text { comida }\end{array}$} & Hombres & 29 & 4.97 & 7.35 & \multirow{2}{*}{.326} & \multirow{2}{*}{0.22} \\
\hline & Mujeres & 57 & 3.60 & 5.32 & & \\
\hline \multirow{2}{*}{$\begin{array}{c}\text { Control de la } \\
\text { familia }\end{array}$} & Hombres & 29 & 6.41 & 4.68 & \multirow{2}{*}{.300} & \multirow{2}{*}{-0.23} \\
\hline & Mujeres & 57 & 7.47 & 4.33 & & \\
\hline \multirow{2}{*}{ Hacer vista gorda } & Hombres & 30 & 1.67 & 2.24 & \multirow{2}{*}{.387} & \multirow{2}{*}{-0.19} \\
\hline & Mujeres & 61 & 2.30 & 3.62 & & \\
\hline \multirow{2}{*}{ TOTAL } & Hombres & 29 & 35.45 & 22.93 & \multirow{2}{*}{.176} & \multirow{2}{*}{-0.31} \\
\hline & Mujeres & 57 & 42.53 & 22.65 & & \\
\hline
\end{tabular}

$\left({ }^{*} \mathrm{p} \leq 0.05 ; * * \mathrm{p} \leq 0.01 ; * * * \mathrm{p} \leq 0.001\right)$. EAISA= Escala de acomodación a los síntomas de enfermedad. 
Así también, en relación al parentesco y al diagnóstico de los pacientes, los análisis mostraron diferencias estadísticamente significativas en la subescala de búsqueda de seguridad, pero los análisis post hoc no identificaron diferencias entre grupos.

Por otro lado, en relación a la edad de los cuidadores los análisis mostraron diferencias estadísticamente significativas en la subescala de rituales en torno a la comida, encontrando que los cuidadores con edades comprendidas entre 20 y 30 años presentaban una menor acomodación frente al resto de cuidadores $(F=5.24, p \leq .001)$.

Continuando con el estudio del malestar emocional, la puntuación media del GHQ-12 fue de $4.58(D T=4.06)$, y encontramos que el $48.3 \%$ de los cuidadores mostraron malestar emocional por encima del punto medio del rango. En relación a esta variable, los análisis no mostraron diferencias significativas en relación al tiempo de evolución del problema, el parentesco, el diagnóstico, ni la edad del cuidador.

En función del género del cuidador, los análisis mostraron diferencias estadísticamente significativas en el malestar emocional, donde las mujeres presentaban puntuaciones medias superiores $(M=5.16 ; D T=4.07)$ que el grupo de los hombres $(M=3.31 ; D T=5.16)($ Ver tabla 5).

En relación a la convivencia, los análisis mostraron diferencias estadísticamente significativas en el malestar emocional, donde los cuidadores que si convivían con las pacientes presentaban puntuaciones medias superiores $(M=4.83 ; D T=4.08)$ que el grupo que no convivían con ellos $(M=1.75 ; D T=2.96)$ (Ver tabla 6$)$.

Tabla 5.- Malestar emocional en el género del cuidador

\begin{tabular}{cccccccc}
\hline & & GÉNERO & $\mathrm{N}$ & MEDIA & $D T$ & $p$ & $d$ \\
\hline \multirow{2}{*}{ GHQ } & Malestar & Hombres & 29 & 3.31 & 3.80 & & \\
& emocional & Mujeres & 63 & 5.16 & 4.07 & & \\
\hline
\end{tabular}

$(* \mathrm{p} \leq 0.05 ; * * \mathrm{p} \leq 0.01 ; * * * \mathrm{p} \leq 0.001) . \mathrm{GHQ}=$ Cuestionario de salud General, GHQ-12.

Tabla 6.- Análisis de diferencias en malestar emocional en la convivencia con el paciente

GÉNERO $\quad \mathrm{N} \quad$ MEDIA $\quad D T \quad p \quad d$

\begin{tabular}{cccccccc}
\hline GHQ & \multicolumn{2}{l}{ Si conviven } & 83 & 4.83 & 4.08 & & \\
$\begin{array}{c}\text { Malestar } \\
\text { emocional }\end{array}$ & $\begin{array}{l}\text { No convi- } \\
\text { ven }\end{array}$ & 8 & & & $.041^{*}$ & 0.76 \\
& & 8.75 & 2.96 & & \\
\hline
\end{tabular}

$(* \mathrm{p} \leq 0.05 ; * * \mathrm{p} \leq 0.01 ; * * * \mathrm{p} \leq 0.001) . \mathrm{GHQ}=$ Cuestionario de salud General, GHQ-12. 
En relación a los análisis de correlaciones entre las variables psicosociales evaluadas, se mostraron correlaciones positivas y estadísticamente significativas entre ellas (Ver tabla 7).

En relación a los análisis de regresión lineal realizados, donde se han utilizado como variables dependientes el malestar emocional, y como independientes el resto de variables psicosociales utilizadas en este estudio, más el tiempo de evolución del problema y el diagnóstico, los análisis mostraron que el $46.5 \%$ de la varianza del malestar emocional fue explicada por el aislamiento social $(\beta=0.68, p<.01) \mathrm{y}$, en menor medida, el $4.6 \%$ de la varianza por la culpa $(\beta=0.51, p<.01)$ (Ver tabla 8$)$.

Tabla 7.- Correlaciones

\begin{tabular}{|c|c|c|c|c|c|c|c|c|c|c|c|c|}
\hline & $\begin{array}{c}\text { GHQ. } \\
\text { TOTAL }\end{array}$ & EDSIS 1 & EDSIS 2 & EDSIS 3 & EDSIS 4 & EDSIS T & EAISA 1 & EAISA 2 & EAISA 3 & EAISA 4 & EAISA 5 & EAISA 6 \\
\hline GHQ.TOTAL & 1 & $.463^{*}$ & $.560^{\circ}$ & $.339^{\prime \prime}$ & .641" & $.635^{*}$ & $.488^{*}$ & $.440^{\circ}$ & $.262^{\circ}$ & $.376^{\circ}$ & $.229^{\circ}$ & $.525^{*}$ \\
\hline EDSIS 1 & & 1 & $.387^{\circ}$ & $.485^{\circ}$ & $.595^{\circ}$ & $.827^{\prime \prime}$ & $.629^{*-}$ & $.528^{\prime \prime}$ & $.332^{\prime \prime}$ & $.635^{\prime \prime}$ & $.440^{\circ}$ & $.709^{*}$ \\
\hline EDSIS 2 & & & 1 & $.503^{*}$ & $.625^{*}$ & $.795^{*}$ & $.442^{*}$ & $.437^{*}$ & $.392^{*}$ & $.299^{*}$ & $.276^{*}$ & $.5322^{*}$ \\
\hline EDSIS 3 & & & & 1 & $.377^{*}$ & $.735^{*}$ & $.335^{*}$ & $.441^{* *}$ & $.398^{* \prime}$ & $.355^{*}$ & $.524^{* *}$ & $.532^{* *}$ \\
\hline EDSIS 4 & & & & & 1 & $.806^{* \prime}$ & $.650^{*}$ & $.633^{*}$ & $.353^{* \prime}$ & $.530^{\circ}$ & $.340^{*}$ & $.725^{*}$ \\
\hline EDSIS T & & & & & & 1 & $.648^{*}$ & .634" & $.467^{*}$ & $.581^{*}$ & $.502^{*}$ & $.7866^{\prime \prime}$ \\
\hline EAISA 1 & & & & & & & 1 & $.555^{\circ}$ & $.299^{*}$ & $.642^{* *}$ & $.415^{* *}$ & $.863^{* *}$ \\
\hline EAISA 2 & & & & & & & & 1 & $.238^{\circ}$ & $.577^{* \prime}$ & $.281^{*}$ & $.769^{\circ}$ \\
\hline EAISA 3 & & & & & & & & & 1 & $.397^{* \prime}$ & $.282^{*}$ & $.586^{\circ}$ \\
\hline EAISA 4 & & & & & & & & & & 1 & $.322^{*}$ & $.802^{*}$ \\
\hline EAISA 5 & & & & & & & & & & & 1 & $.546^{\circ}$ \\
\hline EAISA 6 & & & & & & & & & & & & 1 \\
\hline
\end{tabular}

$\left({ }^{*} \mathrm{p} \leq 0.05 ; * * \mathrm{p} \leq 0.01 ; * * * \mathrm{p} \leq 0.001\right)$. GHQ. TOTAL= Cuestionario de salud General, GHQ-12; EDSIS= Escala de impacto de los síntomas de los trastornos alimentarios; EDSIS 1= Impacto nutricional; EDSIS 2= Culpa; EDSIS 3= Conductas desrreguladas; EDSIS 4= Aislamiento Social, EDSIS T: Puntuación total; EAISA= Escala de acomodación a los síntomas de enfermedad EAISA 1= Evitación y modificación de la conducta; EAISA 2= Búsqueda de seguridad; EAISA 3= Rituales de comida; EAISA 4= Control de la familia; EAISA 5= Hacer la vista gorda; EAISA 6= Puntuación total.

Tabla 8.- Análisis de regresión lineal: Malestar emocional en los cuidadores

\begin{tabular}{ccccc}
\hline Malestar emocional & $\mathrm{R}^{2} / \mathrm{r}$ & Cambio $\mathrm{R}^{2}$ & $\mathrm{~F}$ & $\beta$ \\
\hline Aislamiento Social & & 0.46 & \\
Culpa & $0.51 / 0.71$ & $0.51^{* * *}$ & $7.44^{* * *}$ & \\
& & 0.046 & & $0.27^{* * *}$ \\
\hline
\end{tabular}

$(* \mathrm{p} \leq 0.05 ; * * \mathrm{p} \leq 0.01 ; * * * \mathrm{p} \leq 0.001)$ 


\section{Discusión.}

Este estudio tuvo como objetivo evaluar y describir la acomodación a la enfermedad, la percepción de carga y el malestar emocional en familiares de pacientes con un trastorno alimentario, así como analizar las relaciones entre estas variables.

Respecto a la percepción del impacto de carga, en este estudio empleamos el cuestionario EDSIS, que evalúa el impacto que generan los síntomas del paciente, así como sus comportamientos, directamente en el bienestar de los cuidadores, donde las puntuaciones más altas indican un mayor impacto. Permite obtener una puntuación total, que en nuestro estudio fue de 32.36. Las investigaciones previas revisadas muestran puntuaciones superiores en la percepción del impacto de los síntomas de enfermedad en los cuidadores de pacientes con TA que el obtenido en nuestro trabajo, a excepción del estudio de Girz et al. (2013) realizado en población canadiense (Goddard et al., 2011; Grover et al., 2011; Hibbs et al., 2015; Pepin y King, 2013; Raenker et al., 2013). En los estudios revisados las puntuaciones obtenidas presentan un rango que oscila desde 34 hasta un valor de 46, y encontramos que los estudios realizados con cuidadores españoles suelen presentar puntuaciones más bajas que los estudios con cuidadores británicos. Una posible explicación a esta diferencia cultural es que en los países mediterráneos suele ser más habitual que los hijos pasen más tiempo viviendo junto con la familia, y por tanto, la relación entre el cuidador y la paciente puede dar lugar a que el rol de cuidador sea más llevadero, fácil o tolerante, puntuando con un valor más bajo en esta escala.

Esta percepción del impacto de los síntomas en los cuidadores, podría estar relacionado con el hecho de que vivir con una persona que padece un TA implica convivir con los síntomas que presenta, con su gestión emocional y con las consecuencias de vivir un proceso de enfermedad mental, donde la falta de información que pueden presentar los familiares, y la necesidad de ayuda frente a las consecuencias de la enfermedad (Graap et al., 2008; Haigh y Treasure, 2003), podría implicar una mayor percepción de carga sobre el modo en el que el TA afecta al cuidador. Por otro lado, también puede ocurrir que cuando los síntomas de enfermedad no son comprendidos por los familiares, y en ocasiones, incluso son malinterpretados como un signo de rebeldía o un modo de "llamar la atención" por parte de la paciente, encontremos cuidadores con mayores puntuaciones, ya que les resulta más difícil comprender el TA, su sintomatología, y el malestar que genera.

En relación a la acomodación a los síntomas de la enfermedad, se entiende como el modo en el que la familia se reorganiza alrededor de los síntomas y del TA. La puntuación media de la acomodación total obtenida en este estudio fue de 40.14. La acomodación ha sido señalada como un factor de mantenimiento de los síntomas de enfermedad del TA, y por tanto se ha relacionado con una peor recuperación (Salerno et al., 2016; Treasure y Schmidt, 2013). En el caso de esta investigación, las puntuaciones medias presentan valores inferiores a los aportados por otras investigaciones. Así, por ejemplo Sepúlveda et al. (2009), mostraron que la acomodación presentaba una puntuación media de 49.9. Goddard et al. (2011) obtuvieron puntuaciones que oscilaban entre 47.4 y 45.2. Grover et al. (2011), obtuvieron una puntuación media igual a 47.2. Y Salerno et al. (2016), encontraron una puntuación media de 47.4 . 
Los motivos por los que la familia se acomoda a la enfermedad podrían estar relacionados con la intención de no molestar al paciente con el TA, para reducir discusiones, reacciones emocionales desagradables y para intentar mantener un buen clima familiar. Este intento de ayudar al paciente sin interferir en su TA podría dar más espacio a los síntomas en la vida familiar y por tanto repercutir de manera negativa en el paciente, que tiene más fácil acceder a sus rituales y conductas de enfermedad que al mismo tiempo le hacen empeorar. Por otro lado, también podríamos encontrar mayor acomodación en familias con menor cohesión, o con vidas más independientes, donde los síntomas se permitirían por desconocimiento de que tienen lugar. También hay que tener en cuenta que en ocasiones la familia no es consciente del nivel de acomodación que presenta frente al TA, ya que esas rutinas pueden resultar como cambios sutiles, o puede que lleven tanto tiempo teniendo lugar en el entorno familiar que los cuidadores se han acostumbrado, y por tanto no son tan conscientes. La realidad es que muchas familias cuando están dentro de un proceso de enfermedad como es el TA tienen la sensación de perder la "vida normal de familia" y llegan a dudar de si las actividades que llevan a cabo las realizan como una actividad dentro del marco de una familia con un familiar con TA, o si por el contrario realizan tales actividades como una familia normal. En ocasiones, y por miedo, pierden la confianza en ellos mismos y no tienen claro donde pueden exigir a sus pacientes con TA cambios en sus dinámicas familiares, ya que en ocasiones pueden llegar a dudar de si las conductas del paciente son fruto del TA, fruto de su personalidad, o de la etapa vital que en ese momento les toca vivir, por lo que ese es otro de los motivos por los que la familia puede acostumbrarse o acomodarse a las conductas de enfermedad y permitir que tengan lugar en la vida familiar.

En relación al malestar emocional, encontramos que el $48.3 \%$ de los cuidadores mostraron malestar emocional por encima del punto medio del rango. En otros estudios evaluados con el mismo cuestionario, se ha mostrado que entre el $50.9 \%$ y el $77 \%$ de los cuidadores presentaban puntuaciones elevadas (Berbel et al., 2010; Haig y Treasure, 2003; Winn et al., 2007). El GHQ-12, evalúa entre otras cosas, si el familiar ha sido capaz de tomar decisiones, de centrarse, de tener confianza en sí mismo... como se ha comentado anteriormente nuestros participantes habían sido reclutados de la UTHA, donde también se les ofrecía información y apoyo sobre el TA. Por ello, al sentirse apoyados y reforzados por el equipo de profesionales, es posible que sus puntuaciones fueran inferiores,.

En relación a las diferencias en las variables evaluadas en función de las características sociodemográficas y clínicas de los cuidadores, encontramos que en relación al género se encontraron diferencias significativas entre hombres y mujeres en relación a la percepción de carga en las subescalas de impacto nutricional, culpa, conductas desreguladas, así como, en la acomodación a la enfermedad, en la subescala de búsqueda de seguridad, y en el malestar emocional. Los resultados de acuerdo a la percepción de carga, indican que las mujeres se encontraban más afectadas por la percepción del impacto nutricional de las pacientes, y es que en ocasiones encontramos que dentro de los TA las pacientes niegan la severidad de la situación, así como de los síntomas que presentan, encontrando que de ese modo se puede aumentar el conflicto en la relación entre el cuidador y la paciente (Tan, Hope, Stewart y Fitzpatrick, 2003). 
Así también encontramos que las mujeres del estudio presentaban mayor culpa e impacto por las conductas desreguladas de las pacientes, y los resultados son acordes con la bibliografía revisada. La literatura señala que el apoyo en grupos de cuidadores ayuda a reducir la sensación de aislamiento, soledad, culpa, sobrecarga, malestar emocional y ayuda a mejorar el sentimiento de esperanza de los cuidadores, encontrando también que el riesgo de recaídas de las pacientes se reduce cuando los miembros familiares se ayudan entre sí en la recuperación del trastorno (Holtkamp et al., 2005; Zucker et al., 2005).

Por otro lado, en relación a la acomodación las mujeres presentaban puntuaciones superiores frente a los hombres en la subescala de "búsqueda de seguridad", entendida esta variable como las preguntas recurrentes que las pacientes realizan a los cuidadores para encontrar seguridad en diferentes conductas de enfermedad, como por ejemplo preguntar sobre el valor calórico de los alimentos, sobre la posibilidad de engordar, o mantener conversaciones repetidas sobre diferentes pensamientos o sentimientos negativos que sienten. Es posible que las mujeres del estudio presentaran puntuaciones superiores en esta variable debido a la cercanía en la relación con la paciente con TA, lo que justificaría una mayor relación y cercanía entre ambas.

En relación al malestar emocional, encontramos que las mujeres del estudio presentaban mayor malestar emocional frente a los hombres, una posible explicación surge del planteamiento de que las madres que cuidan de un paciente con TA pueden ser víctimas secundarias del trastorno, ya que internalizan las tensiones y el estrés, y eso repercutiría en su propio bienestar (Kyriacou et al., 2008). La literatura señala que estar al cuidado de una paciente con un TA conlleva una serie de consecuencias negativas, derivadas del cuidado, que influyen de manera directa sobre el malestar emocional de los cuidadores. Además, el malestar emocional es fácil de contagiar, por lo que sería probable encontrar que cuando las pacientes presentasen mayores niveles de malestar, los cuidadores también puntuaran alto en la variable y viceversa. Hemos de tener en cuenta que ser cuidador de un paciente con TA, repercute en el propio rol de cuidador, influyendo y cambiando aspectos de la dinámica personal y familiar diaria, por lo que el cambio de situación, podría afectar de manera más directa a las mujeres, y por tanto presentar mayor malestar emocional por el cambio que el TA crea y supone en sus propias vidas.

Continuando con las diferencias en las variables evaluadas en función de las características sociodemográficas y clínicas de los cuidadores, encontramos que, en relación al tiempo de evolución del problema, el parentesco entre pacientes y cuidadores y el diagnóstico de las pacientes, no se encontraban diferencias significativas. Por otro lado, en relación a la edad de los cuidadores los resultados mostraron diferencias significativas en la percepción de carga en las subescalas de culpa, y en la acomodación en la subescala de "rituales en torno a la comida". En relación a la culpa, encontramos que los cuidadores de más de 60 años presentaban mayor sensación de culpa frente al resto de cuidadores participantes del estudio. Una posible explicación puede venir de que los cuidadores con más edad presentasen un tiempo de evolución de la enfermedad en sus pacientes mayor y por ello se sintieran más culpables por el TA, o bien porque debido a tener una edad mayor, presentaban más dificultades para comprender el TA, y por tanto, se culparan más considerando que "deberían" o "podrían" haber realizado más actos o acciones para ayudar a su familiar con el TA. 
Por otro lado, en relación a la edad de los cuidadores y la acomodación a la enfermedad, encontramos que el rango de edad en el que los cuidadores presentaban menor acomodación hacia los rituales de las pacientes, era en aquellos cuidadores con edades comprendidas entre 20 y 30 años. Considerando que, en nuestro estudio, la participación de las pacientes diagnosticadas con TA estaba en edades comprendidas entre los 10 y los 26 años, podemos entender que los cuidadores con edades más cercanas a los pacientes, probablemente entendiesen mejor a las pacientes por su cercanía en edad y respondiesen mejor a sus síntomas reduciendo por tanto la acomodación a conductas anómalas en relación a la comida.

En relación a la convivencia con la paciente, los resultados mostraron diferencias significativas en el malestar emocional, donde los cuidadores que convivían con las pacientes, presentaban mayor malestar emocional. Este mayor malestar podría estar relacionado con el hecho de que vivir con una persona que padece un TA implica convivir con los síntomas que presenta, con su gestión emocional y con todas las consecuencias de vivir un proceso de enfermedad mental, donde la falta de información que pueden presentar los familiares, y la necesidad de ayuda frente a todas las consecuencias de la enfermedad, (Graap et al., 2008; Haigh y Treasure, 2003), podría implicar un mayor impacto sobre el modo en el que el TA afecta directamente al cuidador, repercutiendo en su malestar emocional.

En relación al estudio de relaciones, encontramos que el aislamiento social predijo un $46.5 \%$ de la varianza del malestar emocional de los cuidadores, y una posible explicación podría ser que este aislamiento social, entendido como la percepción de una mayor presión asociada al cuidado del familiar, menor independencia, más sensación de carga autopercibida, y una menor ayuda, con pérdida de libertad individual, influya en un mayor malestar emocional. Es importante resaltar que ser cuidador de este tipo de pacientes afecta a todos los niveles de la vida familiar como anteriormente hemos mencionado, y en la perdida de libertad individual, los cuidadores pueden sentirse más ansiosos o deprimidos, como consecuencia de las cargas asociadas al cuidado, que desembocaría en un mayor malestar emocional.

Antes de concluir, nos gustaría señalar las limitaciones que presenta nuestro estudio:

Respecto al diseño de esta investigación, destacar que se trata de un estudio transversal, que no permite establecer conclusiones precisas sobre la secuencia temporal de los acontecimientos, y por tanto, no es posible determinar si las características que presentan estos cuidadores, en cuanto a las variables evaluadas, precedieron a la enfermedad de sus pacientes con TCA o son consecuencias de la misma.

Otra limitación es el uso de autoinformes como herramienta para la evaluación del estado de los cuidadores de pacientes con un TA, ya que las respuestas derivadas de cuestionarios, inventarios y escalas se relacionan con el sesgo de deseabilidad social. Aunque el hecho de que el autoinforme se cumplimentase de forma anónima nos lleve a pensar que, si ha ocurrido, haya sido en grado mínimo.

Por otro lado, el hecho de que la totalidad de los participantes procedan de un único recurso hospitalario, reduce la representatividad de todos los cuidadores de pacientes diagnosticados con un TA. 
En último lugar, destacar que la mayoría de cuidadores eran los padres, en concreto las madres de estas pacientes con TA, por lo que proponemos para futuras líneas de investigación que se incluyan grupos de cuidadores representativos en función del parentesco con la paciente.

\section{Referencias}

Berbel, E., Sepúlveda, A. R., Graell, M., Andres, P., Carrobles, J. A., y Morandé, G. (2010). Valoración del estado de salud y psicopatología de los familiares en el trastorno del comportamiento alimentario: diferencias entre cuidadores principales y secundarios. Revista de Psicopatología y Psicología Clínica, 15(3), 79-192.

Birmingham, C., Su, J., Hlynsky, J., Goldner, E., y Gao, M. (2005). The mortality rate from anorexia nervosa. International Journal of Eating Disorders, 38(2), 143-146.

Calvo, R. (2002). Anorexia y Bulimia. Guía para padres, educadores y terapeutas. Barcelona: Planeta.

Carral-Fernández, L., Sepúlveda, A. R., del Barrio, A. G., Graell, M., y Treasure, J. (2013). The Spanish validation of an Eating Disorders Symptom Impact Scale (EDSIS) among caregivers. Psychiatry research, 209(3), 626-631.

Cottee-Lane, D., Pistrang, N., y Bryant-Waugh, R. (2004). Childhood onset anorexia nervosa: The experience of parents. European eating disorders review, 12(3), 169-177.

Girz, L., Lafrance Robinson, A., Foroughe, M., Jasper, K., y Boachie, A. (2013). Adapting family-based therapy to a day hospital programme for adolescents with eating disorders: preliminary outcomes and trajectories of change. Journal of Family Therapy, 35(1), 102-120.

Goddard, E., Macdonald, P., Sepúlveda, A. R., Naumann, U., Landau, S., Schmidt, U., y Treasure, J. (2011). Cognitive interpersonal maintenance model of eating disorders: intervention for carers. The British Journal of Psychiatry, 199(3), 225-231.

Graap, H., Bleich, S., Herbst, F., Trostmann, Y., Wancata, J., y de Zwaan, M. (2008). The needs of carers of patients with anorexia and bulimia nervosa. European Eating Disorder Review, 16, 21-29.

Grover, M., Naumann, U., Mohammad-Dar, L., Glennon, D., Ringwood, S., Eisler, I., ... y Schmidt, U. (2011). A randomized controlled trial of an Internet-based cognitive-behavioural skills package for carers of people with anorexia nervosa. Psychological Medicine, 41(12), 2581-2591.

Haigh, R., y Treasure, J. (2003). Investigating the needs of carers in the area of eating disorders: Development of the Carers's Needs Assessment Measure (CaNAM). European Eating Disorders Review, 11(2), 125-141.

Hibbs, R., Rhind, C., Leppanen, J., y Treasure, J. (2015). Interventions for caregivers of someone with an eating disorder: A meta-analysis. International Journal of Eating Disorders, 48(4), 349-361.

Highet, N., Thompson, M., y King, R. M. (2005). The experience of living with a person with an eating disorder: The impact on the carers. Eating disorders, 13(4), 327-344.

Hjern, A., Lindberg, L., y Lindblad, F. (2006). Outcome and prognostic factors for adolescent female in-patients with anorexia nervosa: 9-to 14-year follow-up. The British Journal of Psychiatry, 189(5), 428-432.

Holtkamp, K., Müller, B., Heussen, N., Remschmidt, H., y Herpertz-Dahlmann, B. (2005). Depression, anxiety, and obsessionality in long-term recovered patients with adolescent-onset anorexia nervosa. European child y adolescent psychiatry, 14(2), 106-110.

Kyriacou, 0., Treasure, J., y Schmidt, U. (2008). Understanding how parents cope with living with someone with anorexia nervosa: Modelling the factors that are associated with carer distress. International Journal of Eating Disorders, 41(3), 233-242.

Padierna, A., Martín, J., Aguirre, U., González, N., Muñoz, P., y Quintana, J. M. (2013). Burden of caregiving among family caregivers of patients with eating disorders. Social psychiatry and psychiatric epidemiology, 48(1), 151-161. 
Pépin, G., y King, R. (2013). Collaborative care skills training workshops: Helping carers cope with eating disorders from the UK to Australia. Social psychiatry and psychiatric epidemiology, 48(5), 805-812.

Perkins, S., Winn, S., Murray, J., Murphy, R., y Schmidt, U. (2004). A qualitative study of the experience of caring for a person with bulimia nervosa. Part 1: The emotional impact of caring. International Journal of Eating Disorders, 36(3), 256-268.

Quiles Marcos, Y., Quiles Sebastián, M. J., Pamies Aubalat, L., Sepúlveda García, A. R., y Treasure, J. (2016). The Spanish Validation of the Accommodation and Enabling Scale for Eating Disorders Among Carers: A Pilot Study. European Eating Disorders Review, 24(1), 62-68.

Raenker, S., Hibbs, R., Goddard, E., Naumann, U., Arcelus, J., y Ayton, A. y Treasure, J. (2013). Caregiving and coping in carers of people with anorexia nervosa admitted for intensive hospital care. International Journal of Eating Disorders, 46(4), 346-354.

Rhind, C., Salerno, L., Hibbs, R., Micali, N., Schmidt, U., Gowers, S., ... y Lo Coco, G. (2016). The Objective and Subjective Caregiving Burden and Caregiving Behaviours of Parents of Adolescents with Anorexia Nervosa. European Eating Disorders Review, 24(4), 310-319.

Rocha, K., Pérez, K., Rodríguez-Sanz, M., Borrell, C., y Obiols, J. (2011). Propiedades psicométricas y valores normativos del General Health Questionnaire (GHQ-12) en población general española. International Journal of Clinical and Health Psychology, 11(1), 125-139.

Salerno, L., Rhind, C., Hibbs, R., Micali, N., Schmidt, U., Gowers, S., ... y Treasure, J. (2016). An examination of the impact of care giving styles (accommodation and skilful communication and support) on the one year outcome of adolescent anorexia nervosa: Testing the assumptions of the cognitive interpersonal model in anorexia nervosa. Journal of affective disorders, 191, 230-236.

Sepulveda, A. R., Kyriacou, 0., y Treasure, J. (2009). Development and validation of the accommodation and enabling scale for eating disorders (AESED) for caregivers in eating disorders. BMC Health Services Research, 9(1), 171.

Stillar, A., Strahan, E., Nash, P., Files, N., Scarborough, J., Mayman, S., ... y Marchand, P. (2016). The influence of carer fear and self-blame when supporting a loved one with an eating disorder. Eating disorders, 24(2), 173-185.

Strober, M. (2004). Managing the chronic treatment-resistant patient with anorexia nervosa. International Journal of Eating Disorders, 36(3), 245-255.

Tan, J. O., Hope, T., Stewart, A., y Fitzpatrick, R. (2003). Control and compulsory treatment in anorexia nervosa: The views of patients and parents. International journal of law and psychiatry, 26(6), 627-645.

Tierney, S. (2005). The treatment of adolescent anorexia nervosa: A qualitative study of the views of parents. Eating Disorders, 13(4), 369-379.

Treasure, J., Smith, G., y Crane, A. (2007). Skills-based Caring for a Loved One with an Eating Disorder: The New Maudsley Method. Routledge.

Treasure, J., y Schmidt, U. (2013). The cognitive-interpersonal maintenance model of anorexia nervosa revisited: a summary of the evidence for cognitive, socio-emotional and interpersonal predisposing and perpetuating factors. Journal of eating disorders, 1(1), 13.

Whitney, J., y Eisler, I. (2005). Theoretical and empirical models around caring for someone with an eating disorder: The reorganization of family life and inter-personal maintenance factors. Journal of Mental Health, 14(6), 575585.

Winn, S., Perkins, S., Murray, J., Murphy, R., y Schmidt, U. (2004). A qualitative study of the experience for caring of a person with bulimia nervosa, Part 2: Carer's needs and experience of services and other support. International Journal of Eating Disorder, 36, 269-279.

Winn, S., Perkins, S., Walwyn, R., Schmidt, U., Eisler, I., Treasure, J., ... y Yi, I. (2007). Predictors of mental health problems and negative caregiving experiences in carers of adolescents with bulimia nervosa. International Journal of Eating Disorders, 4O(2), 171-178.

Zabala, M. J., Macdonald, P., y Treasure, J. (2009). Appraisal of caregiving burden, expressed emotion and psychological distress in families of people with eating disorders: A systematic review. European Eating Disorders Review, $17(5), 338-349$.

Zucker, N. L., Ferriter, C., Best, S. y Brantley, A. (2005). Group parent training: a novel approach for the treatment of eating disorders. Eating Disorders, 13, 391-405. 